\title{
Electron Differential Phase Microscopy with an A-B Effect Phase Plate.
}

\author{
T. Tanji ${ }^{1)}$, H. Niimi ${ }^{2)}$, J. Usukura ${ }^{3)}$, Y. Yamamoto ${ }^{1)}$ and S. Ohta ${ }^{4)}$ \\ ${ }^{1 .}$ EcoTopia Science Institute, Nagoya University, Nagoya, Japan \\ 2. Graduate School of Engineering, Nagoya University, Nagoya, Japan \\ 3. Graduate School of Science, Nagoya University, Nagoya, Japan \\ 4. JEOL Ltd., 3-1-2 Musashino, Akishima, Tokyo, Japan
}

Observations of week phase objects, such as thin films of light elements, thin polymer films, biological sections etc., are available by electron phase microscopy[1]. Many of phase plates utilized are thin film types. Some electrostatic types have been developed[2, 3], but they are not so general, because the fabrication of the filter with fine structures is very difficult. The mainstream of todays phase plate is the thin film type. This type of the phase plate, however, has some disadvantages, i.e. control of the film thickness, charging up, contamination and so on.

We adopted the phase plate with a magnetic thin filament which generates the vector potential around itself by an Aharonov-Bohm (A-B) effect. The filament type phase plate with the A-B effect was proposed and constructed firstly by Nagayama. This type of the phase plate generates the differential phase contrast in the image, and has a longer life time than the thin film type. Any clear differential effect, however, has scarcely reported so far. We will report that the effect of a phase plate consisting of a Wollaston platinum filament less than $1 \mu \mathrm{m}$ in diameter covered with ferromagnetic material, Sm-Co of $5 \mathrm{~nm}$ thick, deposited by a Pules Laser Deposition after demagnetization at $800^{\circ} \mathrm{C}$. The filament with a clean surface selected by SEM is mounted on a single hole $\mathrm{Cu}$ grid, and re-magnetization in the field of $2.4 \mathrm{~T}$.

The phase difference in the both side spaces of the filament measured by electron holography shows 1.5 rad as shown in Fig.1. Being set on the aperture holder, the phase plate is inserted in the back focal plane of the objective. Figure 2 shows images of a mouse photoreceptor cell, which is fixed with Os and without staining. The differential image in Fg.2(b) observed using the phase plate shows inner structures including mitochondria clearer than in the image taken ordinary TEM in Fig.2(a). The direction of the differentiation is shown by the arrowhead.

The effects of the source size and the thickness of the filament on the differentiation will be estimated theoretically, and experiments by a $1000 \mathrm{kV}$ high voltage electron microscope will be reported, too.

\section{Refernce}

[1] K. Nagayama: J. Electronmicrosc., 60 (2011) S43.

[2] H. Boersch: Z. Naturforsh. 2a (11947) 615.

[3] T. Matsumoto and A. Tonomura: Ultramicroscopy, 63 (1996) 5.

[4] The authors acknowledge Prof. T. Kato of Nagoya University and Dr. K. Ozaki of Advanced Industrial Science and Technology for their kind helps for the magnetization of filaments, and financially supported by Grant-in-Aid for Scientific Research 25246001. 

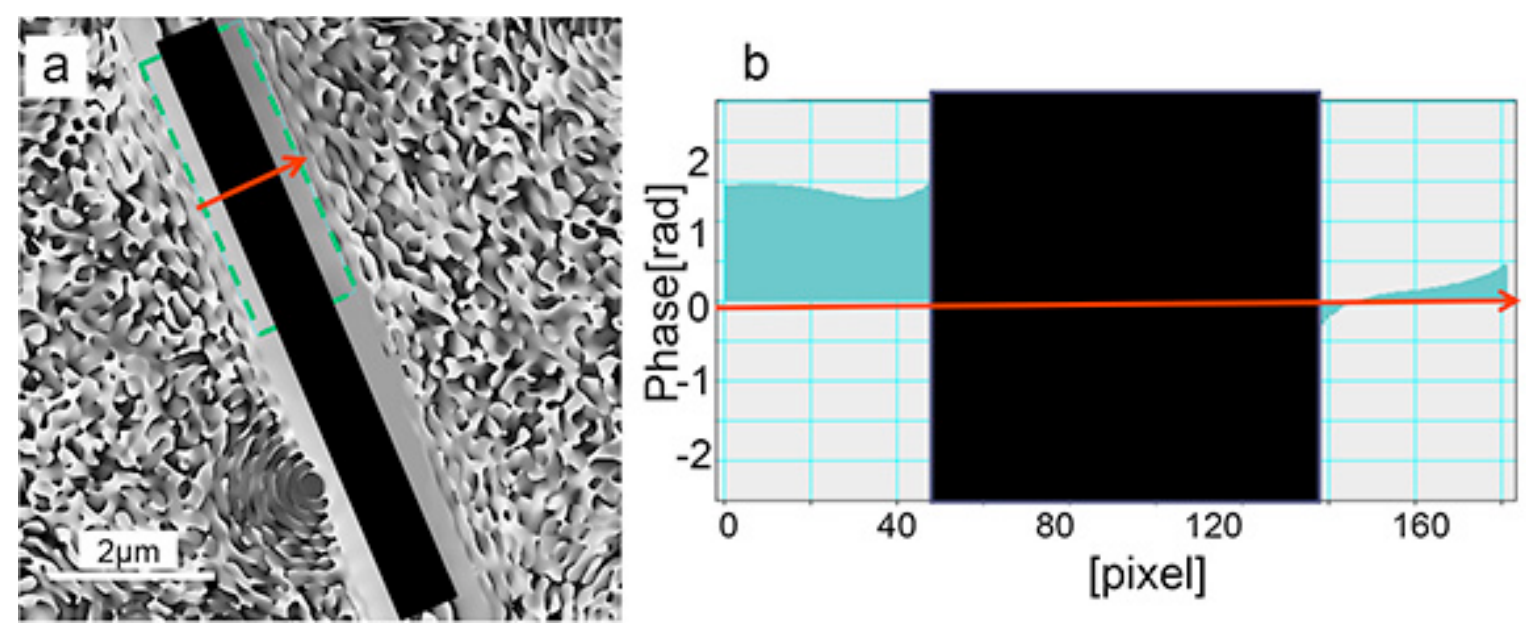

Fig.1 (a) Electron phase map reconstructed by electron holography. (b) A line profile along the arrow head in (a) which is averaged along the long side of the rectangle. The phase difference is about $1.5 \mathrm{rad}$. between both sides of the filament.
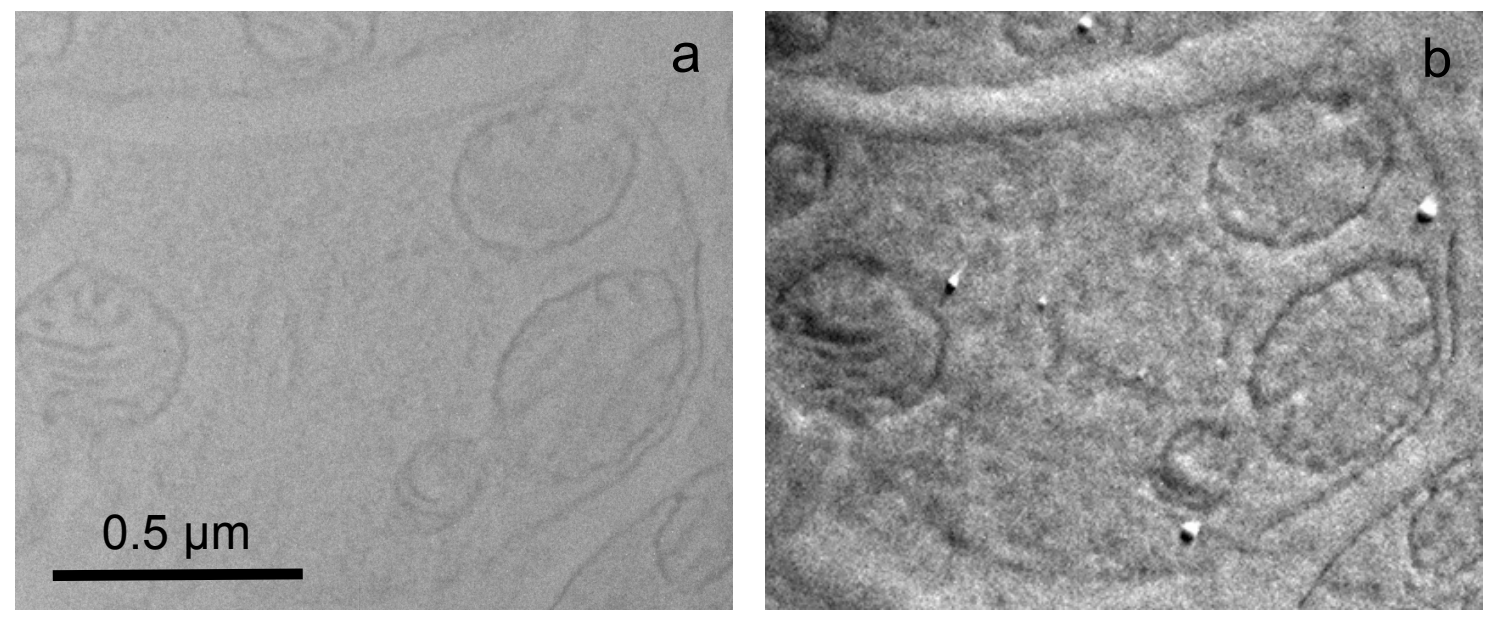

Fig.2 Images of a mouse photoreceptor cell without the phase plate (a), and with the phase plate (b). 